\title{
Eliciting and responding to patient histories of abuse and trauma: challenges for medical education
}

\author{
Toward trauma-informed medical education
}

$\mathrm{T}$

raumatic experiences such as childhood abuse, family violence, elder abuse and combat exposure influence both physical and mental health, health-related behaviour, and the ways in which patients interact with medical practitioners. ${ }^{1,2}$ Despite greater knowledge of the pervasive sequelae of psychological trauma, the implications for medical practice and for medical education are not well articulated. Many doctors lack confidence and remain ill-informed or avoidant when dealing with patients' psychological trauma. ${ }^{3,4}$ The consequences of this include non-recognition of somatisation and of psychiatric disorders, delay in instituting proper treatment, and costs to the patient and health care system of unnecessary investigations and treatments. ${ }^{5,6}$ Here, we discuss why and how we should better train doctors to elicit and respond to patient histories of trauma.

\section{High prevalence of trauma and its clinical sequelae}

The lifetime prevalence of exposure to traumatic events is high (74.9\% in Australian adults). ${ }^{7}$ Most people who experience trauma do not develop mental illness; however, trauma and abuse are substantial contributors to the burden of mental and physical ill health. The risk of post-traumatic stress disorder after trauma is about $10 \%$, but childhood abuse and neglect in combination with later life stress contribute to the development of mental illnesses as diverse as psychoses, depression, eating disorders and addictions, as well as a range of physical illnesses. ${ }^{2,9}$ There are also clear associations between past trauma and abnormal illness behaviour ${ }^{10}$ and, related to this, increased health care use. ${ }^{6}$ These sequelae of patient trauma pervade all medical specialties and also dentistry. ${ }^{1}$

\section{Gabrielle Matta \\ Robyn E \\ Incorporating teaching about trauma in medical curricula: key issues} Woodward-Kron ${ }^{2}$

Sandra Petty

Michael R Salzberg ${ }^{1}$

1 St Vincent's Mental Health, Melbourne, VIC.

2 University of

Melbourne,

Melbourne, VIC

mike.salzberg2@

svha.org.au
Although there are several studies that describe training interventions for specific forms of trauma, $3,11,12$ there is little in the literature on current practices in medical education, either in Australia or elsewhere. The diversity in general structure, content and methods of medical curricula as outlined by the Australian Medical Council (AMC) probably extends to trauma-relevant components. ${ }^{13}$ Despite this diversity, it is possible to offer initial considerations for trauma-informed education. We focus on six interrelated aspects: communication skills; knowledge of the health effects of trauma and abuse; knowledge about the effects of trauma and abuse disclosures on doctors and other health professionals;

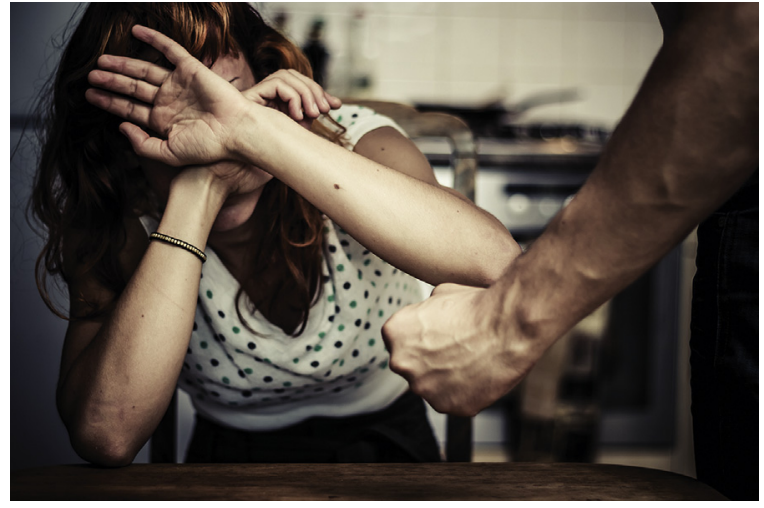

specific knowledge relevant to different medical specialities and settings; teaching formats and methods; and the need for a staged, incremental, integrated program, structured to achieve continuity between undergraduate, prevocational and specialist phases.

Communication skills curricula ${ }^{13}$ in pre-clinical and clinical phases afford opportunities for trauma-specific education, but are also relevant to junior hospital and specialty training. Common issues that need to be addressed include the personal discomfort many doctors experience asking about trauma and abuse; when not to screen for or discuss trauma; and when to seek advice from senior colleagues, as overconfidence can be harmful, leading to patient distress and even re-traumatisation. Communication skills education should extend to discussion of services relevant to different forms of trauma, such as social work, refuges, police and the courts. Here, it would be valuable for medical students to visit these settings or meet with workers from them.

Training needs to be realistic in that doctors often work in settings that are not conducive to asking about trauma, such as busy emergency departments, hospital wards that lack privacy, and overloaded outpatient clinics, often with a lack of psychiatric support. However, these realities should not be a pretext for avoiding clinically competent trauma-informed practice. We do not propose that doctors become trauma therapists; rather that they become competent in empathically recognising and eliciting information about trauma, and at effective referral of patients to relevant services, including psychology and psychiatry.

Exposure to patients who have experienced trauma or abuse evokes a range of psychological reactions in students and trainees, from normal discomfort and distress through to vicarious traumatisation. ${ }^{3,4,14}$ In addition, there may be unhelpful, if not harmful, responses: doctors may adopt an avoidant "don't ask, 
don't tell" style; over-investigate; refer patients to other clinicians; exhibit stigmatising attitudes toward patients; or become over-involved. ${ }^{4}$ Course content on these issues could be introduced early and developed further during clinical and postgraduate phases.

There are many opportunities pre-clinically to learn about the effects of abuse and trauma on human development, including the short and long term effects on the brain and behaviour. Relevant knowledge can be taught within sections of the curriculum devoted to neuroscience, cognitive science and population health. In addition, medical humanities have a powerful capacity to expand our knowledge and understanding of diverse human experiences, including trauma, and to foster empathy. Every clinical specialty that medical students and trainees encounter in hospital training brings opportunities to learn specialty-specific trauma knowledge and skills. For example, rotations in paediatrics and in obstetrics and gynaecology are opportunities for teaching about child abuse and about family violence.

Trauma is an everyday part of clinical discourse within psychiatry and a key dimension of academic and clinical learning in psychiatry rotations. However, trauma-informed education is relevant to all clinical specialties. Relevant specific knowledge encompasses common clinical presentations of trauma in those specialties; how trauma-relevant inquiry can be embedded within the specialty-specific clinical interview; clinical, social and legal services relevant to various forms of abuse; and legal requirements regarding mandatory reporting.

Some medical students and trainees have their own experiences of trauma, including childhood trauma and abuse, but also vicarious trauma stemming from clinical encounters, such as witnessing horrific physical injury or disfigurement. Post-traumatic stress disorder in medical practitioners is often unrecognised. ${ }^{14}$ Such experiences may increase or may impair empathic capacity to engage with traumatised patients.

Although the AMC standards discuss the stressful and traumatic nature of medical work and provide recommendations about availability of counselling, peer support and other measures, they construe the reality of trauma as external to the core business of medical education. ${ }^{13}$ Instead, we propose that learning about the emotional impacts of clinical work should be core medical education, to be dealt with in lectures, tutorials, simulations - that is, a range of appropriate, complementary educational methods, as is done for other topics. In addition, curricula should include safe, confidential, non-coercive opportunities for experiential learning in small groups, allowing participants to reflect on and share their own emotional reactions to patients and understand how these reactions can shape their clinical practice. Ideally, some teaching should be conducted jointly with students from other disciplines, notably nursing.

The AMC standards stress the centrality of clinical clerkships in the development of clinical competence and judgement. ${ }^{13}$ We agree, but when it comes to trauma-informed clinical teaching, there are several entailments. Teaching about trauma has to become a routine, everyday feature of clinical teaching in wards and clinics, not something outsourced by referral to psychiatry or social work. Clinician teachers in all specialties need to acquire the skills to do such teaching and to act as role models. Given the limited evidence base, it is premature to recommend a mix or staging of methods, and this should be a focus of future research and curriculum innovation.

The medical education literature is marked by separate discourses on differing forms of trauma. For example, education regarding intimate partner violence has been extensively explored and excellent curricula have been implemented. ${ }^{3,13,14}$ However, patients have often experienced several concurrent or sequential traumas; and the clinical sequelae of different traumas have many similarities, demand similar clinical skills (albeit allied with different bodies of specific knowledge), and thus present similar challenges for medical education. Valuable educational synergies are likely if the currently disparate, unconnected trauma-relevant elements in medical curricula are integrated.

These considerations point to the need for the creative design and evaluation of staged, incremental, integrated programs, structured to achieve continuity between undergraduate, pre-vocational and specialist phases of medical education. We do not propose removing the various trauma-specific educational components from medical curricula and replacing them with some form of generic trauma education. We do propose, however, examining creatively how they may be better integrated to become mutually reinforcing.

\section{Conclusion}

Trauma-informed health care is an invaluable concept which we propose should extend to trauma-informed medical education. ${ }^{15}$ Although the arguments for trauma-informed medical education are compelling, new lines of educational research will be needed to guide curriculum design and build on the small body of work already available. $3,4,11,12,16$ It is likely that if doctors of all kinds have the knowledge, skills and attitudes to deal competently with abuse and trauma, we can expect improvements in patient care and health service costs, and in the health and wellbeing of medical practitioners. These possibilities deserve empirical study. As well as becoming better clinicians, medical students and trainees will also become better teachers and role models and, as they move into more senior and leadership roles, advocates for competent trauma-informed medical care.

Competing interests: No relevant disclosures.

Provenance: Not commissioned; externally peer reviewed.

(c) 2016 AMPCo Pty Ltd. Produced with Elsevier B.V. All rights reserved.

References are available online at www.mja.com.au. 
1 Raja S, Hoersch M, Rajagopalan CF, Chang P. Treating patients with traumatic life experiences. J Am Dent Assoc 2014; 145: 238-245.

2 Shonkoff JP, Garner AS; Committee on Psychosocial Aspects of Child and Family Health; Committee on Early Childhood, Adoption, and Dependent Care; Section on Developmental and Behavioral Pediatrics. The lifelong effects of early childhood adversity and toxic stress. Pediatrics 2012; 129: e232-e246.

3 Glowa PT, Frasier PY, Newton WP. Increasing physician comfort level in screening and counseling patients for intimate partner violence: hands-on practice. Patient Educ Couns 2002; 46: 213-220.

4 Hendricks-Matthews MK. Ensuring students' well-being as they learn to support victims of violence. Acad Med 1997; 72: 46-47.

5 Lipowski ZJ. Somatization: the concept and its clinical application. Am J Psychiatry 1988; 145: 1358-1368.

6 Gawronski KA, Kim ES, Miller LE. Potentially traumatic events and serious life stressors are prospectively associated with frequency of doctor visits and overnight hospital visits. J Psychosom Res 2014; 77: 90-96.

7 Mills KL, Mcfarlane AC, Slade T, et al. Assessing the prevalence of trauma exposure in epidemiological surveys. Aust N Z J Psychiatry 2011; 45: 407-415.

8 Breslau N, Kessler RC, Chilcoat HD, et al. Trauma and posttraumatic stress disorder in the community: the 1996 Detroit Area Survey of Trauma. Arch Gen Psychiatry 1998; 55: 626-632.
9 McLaughlin KA, Greif Green J, Gruber MJ, et al. Childhood adversities and first onset of psychiatric disorders in a national sample of US adolescents. Arch Gen Psychiatry 2012; 69: 1151-1160.

10 Spitzer C, Barnow S, Gau K, et al. Childhood maltreatment in patients with somatization disorder. Aust N Z J Psychiatry 2008; 42: 335-341.

11 Joyce BL, Jung D, Lucia VC, et al. Developing medical student competence in intimate partner violence: a national priority. Med Sci Educ 2015; 25: 229-232.

12 Lo Fo Wong S, Wester F, Mol SS, Lagro-Janssen TL. Increased awareness of intimate partner abuse after training: a randomised controlled trial. $\mathrm{Br} J \mathrm{Gen}$ Pract 2006; 56: 249-257.

13 Australian Medical Council. Standards for assessment and accreditation of primary medical programs by the Australian Medical Council 2012. AMC: Canberra, 2012. http://www.amc.org.au/accreditation/primary-medicaleducation\#primary-medical-education-procedures (accessed May 2016).

14 Lazarus A. Traumatized by practice: PTSD in physicians. J Med Pract Manag 2014; 30: 131-134.

15 Machtinger EL, Cuca YP, Khanna N, et al. From treatment to healing: the promise of trauma-informed primary care. Womens Health Issues 2015; 25: 193-197.

16 Brandt EN Jr. Curricular principles for health professions education about family violence. Acad Med 1997; 72(1 Suppl): S51-S58. 